\title{
The effect of pegylated granulocyte colony-stimulating factor treatment prior to experimental mastitis in lactating Holsteins
}

\author{
E. J. Powell, ${ }^{*} \dagger$ T. A. Reinhardt, ${ }^{*}$ E. Casas, ${ }^{*}$ and J. D. Lippolis ${ }^{* 1}$ \\ *Ruminant Diseases and Immunology Research Unit, USDA Agricultural Research Service, National Animal Disease Center, Ames, IA 50010 \\ †Oak Ridge Institute for Science and Education, Oak Ridge Associated Universities, Oak Ridge, TN 37830
}

\begin{abstract}
Neutrophils are the first-acting and most prominent cellular defense against mastitis-causing pathogens. This makes neutrophil activation and expansion obvious candidates for targeted therapeutics. The granulocyte colony-stimulating factor (G-CSF) cytokine stimulates the bone marrow to produce granulocytes and stem cells and release them into the bloodstream, which results in neutrophilia as well as increasing the presence of other progenitor cells in the bloodstream. A pegylated form of G-CSF (PEG-gCSF) has been shown to significantly decrease naturally occurring mastitis rates in cows postpartum. The use of PEG-gCSF had not been evaluated in response to an experimental mastitis challenge. In an effort to examine the effect and mechanism of PEG-gCSF treatment, we challenged 11 mid-lactation Holsteins with $\sim 400$ cfu Escherichia coli $\mathrm{P} 4$ by intramammary infusion. Five cows received 2 PEG-gCSF injections, one at $14 \mathrm{~d}$ and the other at 7 $\mathrm{d}$ before disease challenge, and 6 cows remained untreated. To evaluate the response of cows to the PEGgCSF treatment, we measured complete blood counts, somatic cell counts, bacterial counts, milk yield, and feed intake data. The PEG-gCSF-treated cows had significantly increased circulating levels of neutrophils and lymphocytes after each PEG-gCSF injection, as well as following mastitis challenge. The PEG-gCSFtreated cows had significantly lower bacterial counts and lower milk BSA levels at the peak of infection. In addition, control cows had significant decreases in milk yield postinfection and significantly reduced feed intake postinfection compared with PEG-gCSF-treated cows. Collectively, PEG-gCSF treatment resulted in reduced disease severity when administered before a bacterial challenge. Mechanistically, we show that G-CSF treatment increases cell surface expression of an E-selectin ligand before infection on neutrophils and monocytes
\end{abstract}

Received February 6, 2018.

Accepted April 24, 2018.

${ }^{1}$ Corresponding author: john.lippolis@ars.usda.gov found in the blood. These cells quickly disappear from the blood shortly after infection, suggesting a mechanism for the reduced mastitis severity by priming immune cells for quick targeting to the site of infection. Key words: mastitis, cytokine, granulocyte colonystimulating factor, immunology

\section{INTRODUCTION}

Mastitis is the primary health and economic concern for the dairy industry. In dairy cows, mastitis can result in culling, milk loss, reduced milk quality, increased treatment costs (Ryman et al., 2015; Ceniti et al., 2017 ) and is responsible for an estimated $\$ 2$ billion/ yr in loss to the industry in the United States, generally varying costs between $\$ 95$ to $\$ 211$ per case per cow (USDA-APHIS, 2007; Cha et al., 2011; Ryman et al., 2015; Ceniti et al., 2017). Mastitis affects all dairy livestock and can be caused by a plethora of microorganism pathogens, including most commonly in bovine cases Escherichia coli, Staphylococcus aureus, and Streptococcus uberis (Ryman et al., 2015; Ismail, 2017). Symptoms of mastitis can include udder swelling, heat, hardness, or redness; animals may be lethargic or have changes in milk composition and quality, and show losses in milk production and feed intake (Yeiser et al., 2012). Mastitis can vary in levels of severity that can be influenced by environmental and genetic components unrelated to disease challenge (Mallard et al., 1998; Burvenich et al., 2007).

Neutrophils, as well as additional innate effector cells such as monocytes, are activated during normal inflammation responses to infections, and are considered to be the primary innate responders to mastitis infection (Kehrli et al., 1991a). Neutrophils have various killing mechanisms to destroy pathogens (Paape et al., 2003; Brinkmann et al., 2004; Lippolis et al., 2006). Upon encountering invading bacteria, neutrophils can ingest the bacteria into phagosomes that fuse with lysosomes. This process stimulates neutrophils to produce large amounts of oxidizing agents in a process referred to as respiratory burst, which generates various antimicro- 
bial oxidants that kill pathogens, and is also negatively correlated with mastitis disease severity (Heyneman et al., 1990). Last, neutrophils can secrete genomic DNA bound with antimicrobial proteins to physically trap and destroy pathogens (Brinkmann et al., 2004).

Myeloperoxidase (MPO) has long been known to be important to neutrophil function because of its role in the generation of antimicrobial oxidants (Paape et al., 2003; Silvescu and Sackstein, 2014). Periparturient immune suppression and mastitis susceptibility is associated with decreased neutrophil function and is speculated to be a contributor to reduced immune health especially in the first few weeks postpartum (Kehrli et al., 1991b; Mallard et al., 1998; Revelo et al., 2011). Enzymatic function and expression of MPO have been shown to be reduced during the periparturient period (Cai et al., 1994; Lippolis et al., 2006). Recently, MPO has been shown to be a cell surface activation marker on both neutrophils and monocytes in a uniquely glycosylated form that can act as a ligand for E-selectin, which is important for leukocyte migration to areas of inflammation (Silvescu and Sackstein, 2014).

Immune cell regulation for the treatment and prevention of mastitis is a developing field for dairy health therapy. Although a variety of commercial and herdspecific vaccines exist, they are not cost efficient or adequately effective to control all mastitis (Ismail, 2017). While mastitis preventative vaccines remain limited in their efficacy, the most common course of mastitis treatment is antibiotics. Concerns over selecting antibiotic resistant strains of bacteria is the major reason for the search for antibiotic alternatives. Nonantibiotic alternative therapies are under development that include immune stimulators designed to regulate immune cells important to mastitis defense, with an emphasis on neutrophils (Canning et al., 2017; McDougall et al., 2017). One such way to mediate the immune response is the administration of cytokines, such as granulocyte colony-stimulating factor (G-CSF).

The G-CSF is well established as a regulator of myeloid cells. Although evidence indicates that G-CSF is produced by several immune cell sources, it is well established to be produced by monocytes and macrophages in response to inflammatory signaling (Sieff, 1987; Kehrli et al., 1991a; Xu et al., 2000). Granulocyte colony-stimulating factor induces proliferation of myeloid progenitor cells from the bone marrow but also has been shown to stimulate granulocyte maturation and activate circulating mature myeloid cells such as neutrophils (Sieff, 1987; Kehrli et al., 1991a; Xu et al., 2000). As a therapeutic, G-CSF is used in human medicine to recover depleted immune cells (Xu et al., 2000; Silvescu and Sackstein, 2014) and in veterinary health practices to initiate neutrophilia (Kimura et al., 2014; Silvescu and Sackstein, 2014). Recombinant bovine GCSF supplementation has been shown to significantly increase circulating leukocytes including increased numbers of mature and immature band neutrophils (Harp et al., 1991; Kehrli et al., 1991b; Stabel et al., 1991). A pegylated G-CSF (PEG-gCSF) bovine treatment is commercially available (Imrestor/pegbovigrastim, Elanco, Greenfield, IN) with a utilization emphasis on mastitis prevention during the periparturient critical period. In previously reported work, a 2-injection treatment of PEG-gCSF prepartum (first injection approximately $1 \mathrm{wk}$ before calving and second injection day of calving) has been shown to significantly decrease the incidence of naturally occurring mastitis up to 30 d postpartum compared with untreated control cows (Ruiz et al., 2017). This 2-step PEG-gCSF treatment induced increased circulating numbers of monocytes, lymphocytes, and targeted expansion of neutrophils (McDougall et al., 2017).

Exploring the mechanism of neutrophil differentiation, activation, and migration will continue to be important in development of mastitis therapeutics. The objective of this study was to assess for the first time the effect of PEG-gCSF on experimental mastitis and provide early insight into some of the mechanisms of a G-CSF immune response.

\section{MATERIALS AND METHODS}

\section{Experimental Design}

Eleven mid-lactation Holsteins averaging $141.3 \pm$ 20.6 DIM at the start of the experiment were used for this study. Five cows received subcutaneous injections of PEG-gCSF 14 and $7 \mathrm{~d}$ before disease challenge (2 injections total); each injection consisted of $15 \mathrm{mg}$ of PEG-gCSF in $2.7 \mathrm{~mL}$ in a single-dose syringe (Imrestor, Elanco). Six cows remained as untreated controls. Previous experience with our experimental mastitis model demonstrated that a group size of 5 cows could yield significant differences by treatment (Lippolis et al., 2011). Of the experimental animals, 9 were multiparous and 2 were primiparous. The 2 primiparous cows were randomly balanced between control and treatment groups. All 11 lactating Holsteins were challenged with $\sim 400$ cfu E. coli P4 by intramammary infusion in a single quarter, which were clean before the trial start as determined by bacterial culture (data not shown). Feed intake, milk yield, bacterial counts, BSA levels in milk, and DNA in milk will be used as metrics to determine infection severity. Feed intake and total milk yield were recorded daily in addition to relevant blood 
and milk experimental samples. All animals were maintained at the National Animal Disease dairy facility. All protocols used were approved by the National Animal Disease Center Animal Care and Use Committee and comply with guidelines and accepted practices for the care and use of large animals in research.

\section{SCC}

Milk samples were aseptically collected from the experimental quarter on experimental $\mathrm{d}-20,-18$, $-14,-12,-10,-7,-5,-3$ and 0 before challenge to confirm infection-free status as well as postinfection on d 0.25 ( $6 \mathrm{~h}$ postchallenge), 0.5 (12 h postchallenge), 1 , $1.5,2,2.5,3,3.5,4,7,9$, and $11 \mathrm{~d}$ postchallenge. Milk samples were shipped to Dairy Lab Services (Dubuque, IA) for determination of SCC values.

\section{Bacterial Counts}

Milk samples were aseptically collected from the single experimental quarter, serially diluted, and 100 $\mu \mathrm{L}$ of sample was spread across a MacConkey agar plate (catalog no. 211387, BD Biosciences, San Jose, $\mathrm{CA})$. Plates were incubated at $37^{\circ} \mathrm{C}$ overnight and the number of colonies was counted.

\section{BSA ELISA}

The BSA levels were measured in skim milk samples from the experimental quarter with a BSA ELISA (catalog no. E11-113, Bethyl Laboratories, Montgomery, TX). Samples were diluted 1:50,000 and measured in duplicate with a plate reader at $450 \mathrm{~nm}$ per kit instructions (FlexStation3, Molecular Devices, Sunnyvale, CA). For our laboratory, the interassay coefficient of variation was $15.2 \%$, and intraassay coefficient of variation was $5.9 \%$.

\section{Complete Blood Counts and Hematology Slides}

Complete blood counts (CBC) were completed in house by a technician or veterinarian using an automated CBC machine (VetScan Hm5, Abaxis, Union City, CA). Blood smears were mounted and stained from whole blood collected via jugular venipuncture into tubes containing EDTA. Sample identifications were blinded for evaluation. A total of 100 cells were characterized from blood slides in which the proportion of band and mature neutrophils were used to calculated cell numbers from total neutrophil counts from the automated CBC total cell numbers.

\section{Neutrophil Extracellular Trap Analysis}

One hundred milliliters of raw milk from the experimental quarter was spun at $10,000 \times g$ for $45 \mathrm{~min}$ at $4^{\circ} \mathrm{C}$ in $50-\mathrm{mL}$ conical tubes. Milk fat samples were scraped off the top of tubes and washed once with PBS supplemented with protease inhibitor (5 tablets/L of SigmaFast Protease Inhibitor, catalog no. S8830, SigmaAldrich, St. Louis, MO). Milk fat samples were frozen at $-20^{\circ} \mathrm{C}$ until experiment trial period was complete for all cows. Samples were then thawed for analysis by experimental day. Thawed samples were stained with 5 $\mu M$ Sytox Orange (catalog no. S11368, Invitrogen, Eugene, OR) for $15 \mathrm{~min}$ at room temperature and washed once at $4^{\circ} \mathrm{C}$ with neutrophil extracellular traps (NET) buffer $[10 \mathrm{~m} M$ Tris, $150 \mathrm{mM} \mathrm{KCl,} 250 \mathrm{~m} M$ sucrose, 2 $\left.\mathrm{m} M \mathrm{MgCl}_{2}(\mathrm{pH}=7.5)\right]$. Samples were resuspended in NET buffer and evaluated in duplicate on a plate reader at $570 \mathrm{~nm}$ (FlexStation3, Molecular Devices) and by confocal microscopy. Paired controls were first treated with DNase (1 unit per $10 \mathrm{mg}$ of milk fat; catalog no. E8263-25KU, Benzonase, Sigma-Aldrich) for $30 \mathrm{~min}$ at room temperature (Reinhardt et al., 2013). The NET mean fluorescent intensity (MFI) is calculated within animal as $($ NET MFI $)=($ raw sample MFI $)-($ DNase sample MFI). For confocal microscopy imaging, stained samples and DNase controls were normalized and imaged using a Nikon A1R+ laser scanning microscope and the NIS-Elements imaging software (Nikon Instruments, Melville, NY). All slide depictions are $20 \times$ objective, 75 numerical aperture, and were imaged using a GaASP detector (Nikon Instruments), 561 laser.

\section{Flow Cytometry}

Whole blood was stained with CD14 (catalog no. 301808, BioLegend, San Diego, CA), CD45 (catalog no. BOV2039, Monoclonal Antibody Center, Washington State University), and MPO (catalog no. VPA00193, Bio-Rad, Hercules, CA) marker antibodies. Primary antibodies, secondary antibodies, and directly conjugated antibodies were independently incubated for 15 min in the dark, washing between each step with cell staining buffer (catalog no. 420201, BioLegend). Counting beads (catalog no. ACBP-100-10, Spherotech AccuCount Particles, Lake Forest, IL) were added to facilitate calculation of cell numbers, and samples were treated for $30 \mathrm{~min}$ with lysing solution (catalog no. 349202, FACS Lysing Solution, San Jose, CA). Samples were run on a Becton Dickinson LSR II flow cytometer and evaluated using the FlowJo software (FlowJo LLC, Ashland, OR). 


\section{Gating Strategy}

All cells analyzed were live, single-cell CD45+ populations. Monocytes were gated based on forward and side scatter and were CD14+. The neutrophil population was gated with forward and side scatter for granulocytes. Then, within the granulocyte population, auto-fluorescing gating was used to remove eosinophils (Dorward et al., 2013). Neutrophil and monocyte populations were evaluated individually for MPO expression.

\section{Statistical Analysis}

Multiple linear regression models were fitted in $\mathrm{R}$, featuring fixed effects of treatment and either experimental day or experimental phase, respectively. Analysis of variance tables were evaluated for significance of the interaction of PEG-gCSF treatment by day/phase. Specific contrasts of interest were evaluated with a pairwise comparison of the least squares means (LSM) estimates between treated and untreated groups for certain experimental time points (days or phases). Significance denotes $P$-values $\leq 0.05$. All error bars represent standard errors.

Bacterial Load Analysis. Bacterial counts were base $_{10}$ log-transformed. The PEG-gCSF treatment and experimental day were fitted as fixed effects and evaluated for significance. The interaction of PEG-gCSF treatment by day and the LSM contrasts for PEGgCSF-treated and untreated groups were evaluated for significance.

SCC Analysis. The SCC numbers were base ${ }_{10} \log$ transformed. Phase of experiment [preinfection (d -20 to 0 before challenge), or postinfection (d 0 to 11 after challenge)] was evaluated as a fixed effect and the interaction of phase by PEG-gCSF treatment was evaluated for significance. Least squares means contrasts for PEG-gCSF-treated and untreated groups were evaluated for differences within phases.

Milk Yield and Feed Intake. For analysis of milk yield and feed intake, phase of experiment [preinfection ( $d-14$ to -2 before challenge), feed change ( $d-1$ and -2 ), or postinfection (d 0 to 11 after challenge)] was evaluated as a fixed effect, and the interaction of phase by PEG-gCSF treatment was evaluated for significance. Feed intake percentage was based on the feed consumed by each animal daily, divided by the average feed consumed for $7 \mathrm{~d}$ before the start of the experiment. The LSM contrasts for PEG-gCSF-treated and untreated groups were evaluated for differences within phases.

BSA and NET Analysis. The PEG-gCSF treatment and experimental day were fitted as fixed effects and evaluated for significance. The interaction of PEGgCSF treatment by day and the LSM contrasts for
PEG-gCSF-treated and untreated groups were evaluated for significance.

$\boldsymbol{C B C}$ Analysis. Cell subset counts were evaluated separately for total neutrophils, monocytes, and lymphocytes. Within total neutrophils, percentage of band versus mature neutrophil differentiation was done by hand by a trained technician via stained blood slide analysis and adjusted counts were analyzed. The PEGgCSF treatment and experimental day were fitted as fixed effects and evaluated for significance. The interaction of PEG-gCSF treatment by day and the LSM contrasts for PEG-gCSF-treated and untreated groups were evaluated for significance.

\section{RESULTS}

Infections were confirmed in all cows; bacteria was isolated from all infected cows for at least 2 time points (data not shown). All cows had a mild clinical infection that resulted in minimal to no fever and minor to moderate effects on milk quality. For SCC, milk yield, and feed intake, data were analyzed at all stages of the study. We evaluated data grouped into phases of preinfection ( $\mathrm{d}-14$ to -2 before challenge), postinfection (d 0 to 11 after challenge), and feed change ( $d-1$ and $-2)$, which captures an unavoidable change in silage being fed to experimental cows (SCC data were not collected during the feed change phase).

Raw SCC data are shown in Figure 1A, whereas logtransformed SCC are shown in Figure 1B. The PEGgCSF treatment did not result in a difference in SCC $\left(85.6 \times 10^{3} \pm 20.2 \times 10^{3}\right.$ SCC $)$ compared with control cows $\left(21.8 \times 10^{3} \pm 3.9 \times 10^{3} \mathrm{SCC}\right)$ during the preinfection phase for raw SCC (Figure 1A). However, if the SCC is log-transformed, PEG-gCSF cows had significantly higher $(P<0.01)$ SCC $\left[4.5 \pm 0.1 \log _{10}(\mathrm{SCC})\right]$ than nontreated cows $\left[4.2 \pm 0.1 \log _{10}(\mathrm{SCC})\right]$ in the preinfection phase. No differences were detected between PEG-gCSF-treated and nontreated cows during the postinfection phase for raw or log-transformed data.

Milk yield and feed intake were evaluated daily for all cows (Figure 1C, 1D). No differences in milk yields were discernable before infection between PEG-gCSFtreated $(32.8 \pm 0.9 \mathrm{~kg} / \mathrm{d})$ and control cows $(34.1 \pm$ $0.8 \mathrm{~kg} / \mathrm{d}$; Figure 1C). During the feed change phase, PEG-gCSF-treated cows $(28.7 \pm 2.1 \mathrm{~kg} / \mathrm{d})$ did not have significantly different milk production than the untreated animals $(24.6 \pm 1.9 \mathrm{~kg} / \mathrm{d} ; P=0.15)$. However, milk yields for treated cows $(29.9 \pm 1.1 \mathrm{~kg} / \mathrm{d})$ and nontreated control cows $(26.7 \pm 1.0 \mathrm{~kg} / \mathrm{d})$ were statistically different $(P=0.03)$ postinfection. For feed intake (Figure 1D), no difference was observed in feed consumed between PEG-gCSF-treated cows (94.1 \pm $2.4 \%)$ and untreated cows $(88.4 \pm 1.9 \%)$ for the pre- 

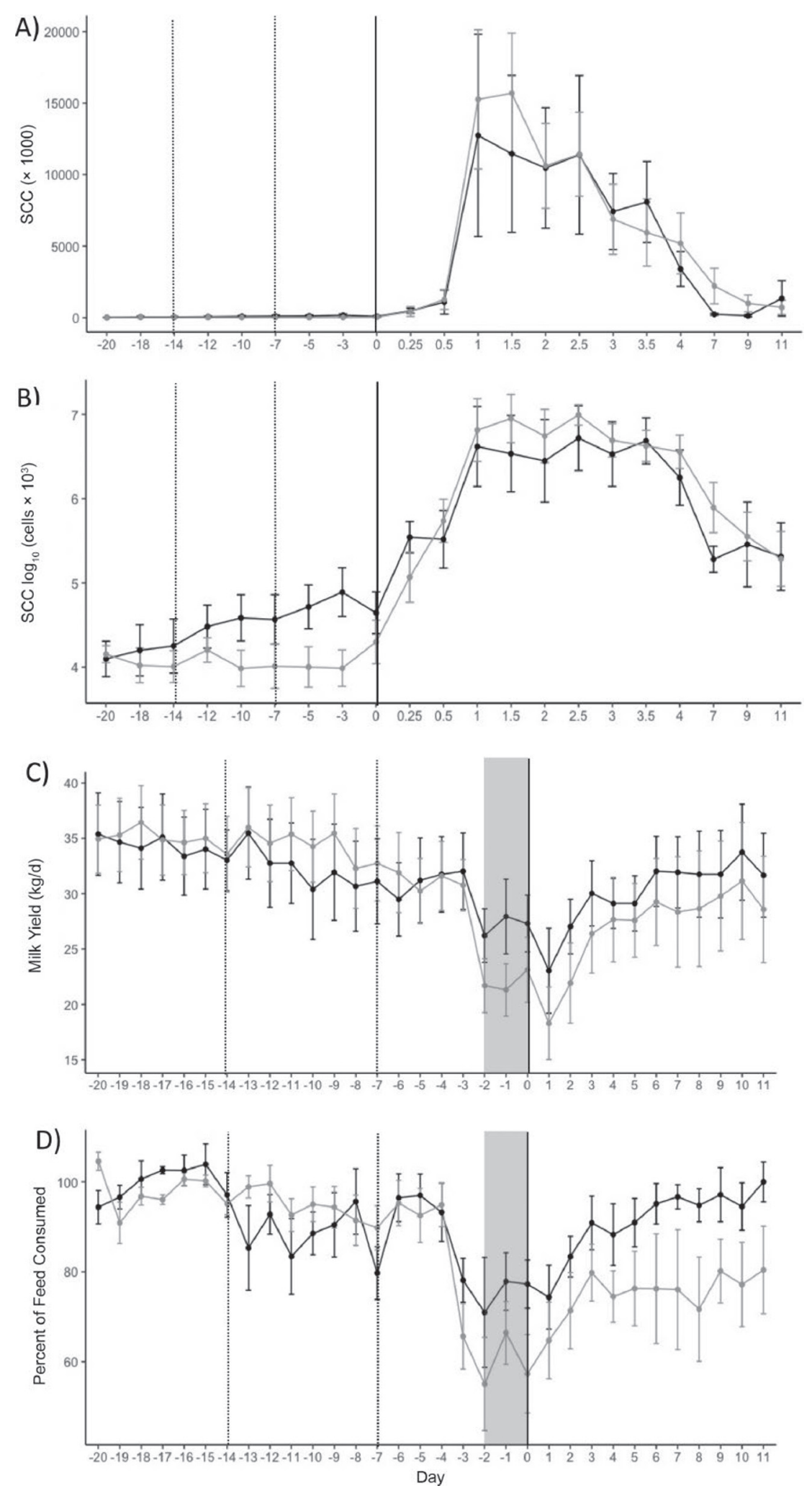

Figure 1. Effect of pegylated granulocyte colony-stimulating factor (PEG-gCSF) therapy (black) on raw and transformed SCC, milk yield, and percent feed intake compared with control cows (gray). (A) Raw SCC, (B) log(base 10 )-transformed SCC, (C) daily milk yield (2 milkings per day), and (D) percentage feed intake (percent consumed of pre-experiment feed offered). Vertical dashed lines indicate PEG-gCSF treatment injections, solid vertical line indicates start of disease challenge, and gray areas denote the silage feed change adjustment period before challenge. Error bars represent SE. 
infection phase. During the feed change phase, PEGgCSF-treated cows ate a higher percentage of offered feed $(75.5 \pm 5.6 \%)$ compared with $(58.8 \pm 4.6 \%)$ by control cows $(P=0.02)$. Postinfection, PEG-gCSFtreated cows consumed a higher percentage of feed $(90.0 \pm 2.8 \%)$ than the untreated control cows $(69.9 \pm$ $2.3 \% ; P<0.01)$.

Bacterial counts were determined for milk samples obtained at indicated time points postinfection (Figure 2A). The PEG-gCSF-treated cows had a significantly decreased bacterial load at $24 \mathrm{~h}(P<0.01), 36 \mathrm{~h}(P=$ $0.01)$, and $48 \mathrm{~h}(P<0.01)$. As a means to assess disease severity, we examined BSA levels of skim milk and found a significant effect of PEG-gCSF treatment $(P$
$=0.05$; Figure 2B). Specifically, PEG-gCSF cows had reduced BSA levels $(3.1 \pm 0.7 \mathrm{mg} / \mathrm{mL}) 24 \mathrm{~h}$ postchallenge $(P<0.01)$ compared with untreated cows $(7.0$ $\pm 0.7 \mathrm{mg} / \mathrm{mL}$ ). Although not statistically significant, treated animals additionally had a lower rectal temperature immediately postinfection (data not shown).

We analyzed whole blood to determine specific behavior of responding immune cell populations and analyzed blood slides to determine the abundance of mature and progenitor neutrophils (Figure 3). The CBC showed PEG-gCSF-treated cows had significantly increased circulating levels of neutrophils after each PEG-gCSF injection $(P<0.01)$. Circulating neutrophil counts remained significantly higher in PEG-gCSF-treated cows
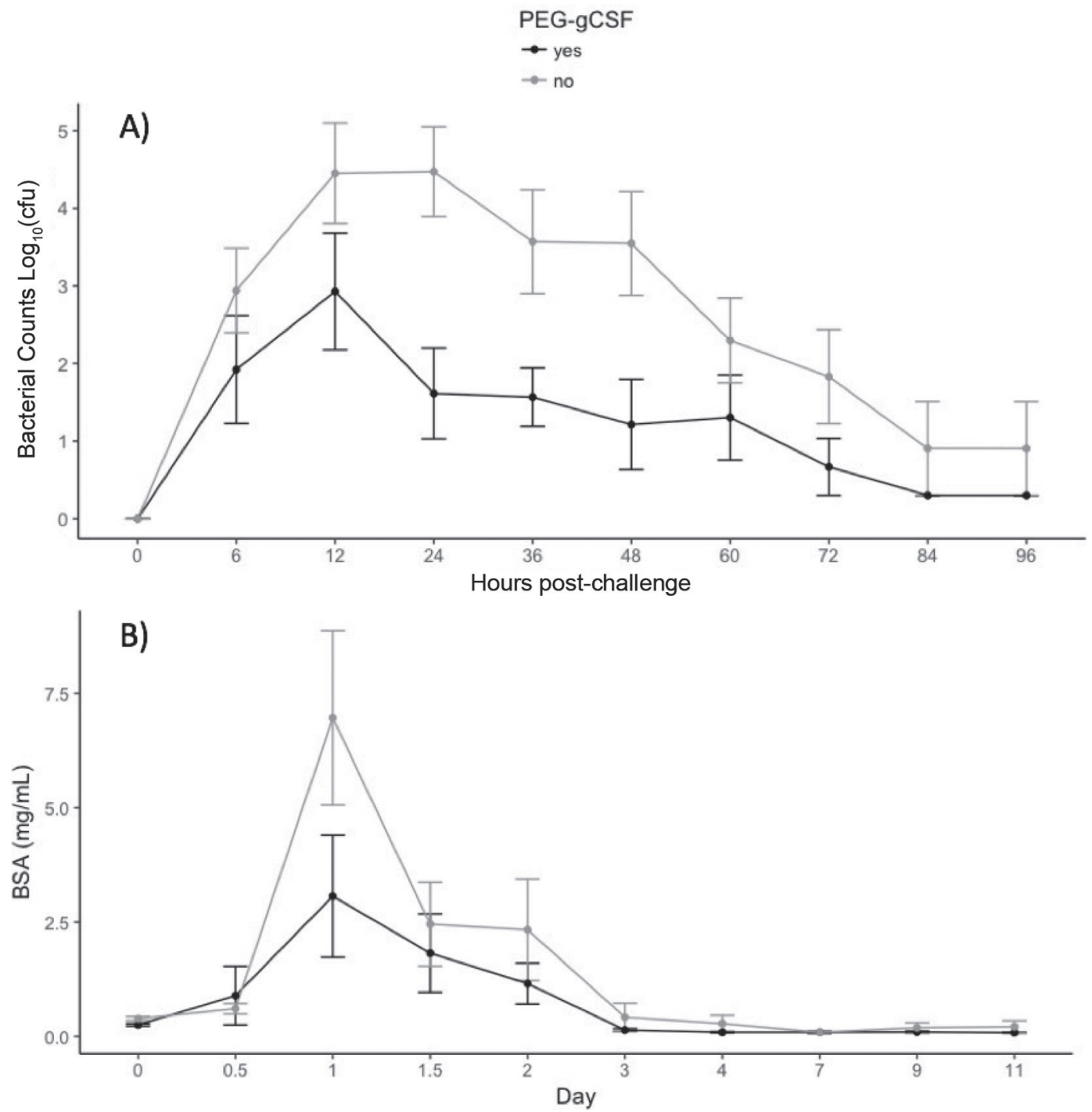

Figure 2. Pegylated granulocyte colony-stimulating factor (PEG-gCSF)-treated cows show reduced severity of disease after mastitis challenge. (A) Log-transformed bacterial counts $\left[\log _{10}(\mathrm{cfu})\right]$ and (B) BSA levels $(\mathrm{mg} / \mathrm{mL})$ were lower for PEG-gCSF-treated cows compared with control cows. Error bars represent SE. 
after the first PEG-gCSF injection until $7 \mathrm{~d}$ postchallenge $(P \leq 0.01$; Figure $3 \mathrm{~A})$. Neutrophil numbers in the blood peaked $4 \mathrm{~d}$ after the first and dramatically $2 \mathrm{~d}$ following the second injection (Figure 3A). Whole blood slides were generated to analyze the proportion of cells that were immature band neutrophils compared with mature, established neutrophils. By adjusting CBC counts to the percentages of band and mature neutrophils, we discovered that in the PEG-gCSF-treated cows, the mature neutrophils are the first responders to the initial PEG-gCSF injection (Figure 3B) beginning $2 \mathrm{~d}$ postinjection $(\mathrm{d}-12)$. The immature (band) neutrophils appear in circulation $4 \mathrm{~d}$ after the first PEGgCSF injection (Figure 3C; $\mathrm{d}-10$ ), and together these compose the initial neutrophil peak identified in Figure 3A. By contrast, immature neutrophils do not appear in the blood of untreated cows until $24 \mathrm{~h}$ postchallenge, where levels are not significantly different from treated cows $(P=0.38)$. Also, monocytes saw a significant increase in circulating numbers $4 \mathrm{~d}(\mathrm{~d}-10)$ after the first PEG-gCSF injection in treated cows $(P<0.01$; Figure 3D). Lymphocytes responded significantly to PEG-gCSF treatment $(P<0.01)$, equally increasing circulating numbers to both PEG-gCSF treatments; 4 $\mathrm{d}$ after the first injection and $2 \mathrm{~d}$ following the second injection $(P<0.01$; Figure $3 \mathrm{E})$.

Next, we analyzed milk fat NET to examine neutrophil activity in milk (Figure 4). We showed increased DNA in milk fat by staining with the DNA stain Sytox Orange. Fluorescence intensity of milk fat samples were quantified on a plate reader (Figure 4A) and characterized with confocal microscopy (Figure 4B). No significant differences exist between PEGgCSF-treated and control cows at experimental d 0 $(P=0.13)$; however, the PEG-gCSF-treated cows had higher NET MFI $24 \mathrm{~h}$ postchallenge $(P=0.02)$, as well as showing larger NET globules under confocal examination (Figure 4B).

To investigate the activation status of immune responders in circulating blood, we stained circulating neutrophils (Figure 5) and monocytes (Figure 6) for surface expression of MPO. Increased surface expression of MPO was evident in both neutrophils (Figure 5B) and monocytes (Figure 6B) after both PEG-gCSF injections in treated cows compared with no detectable differences pretreatment (Figure 5A, 6A). Interestingly, the populations of "activated" neutrophils and monocytes disappear in whole blood $24 \mathrm{~h}$ postchallenge in PEG-gCSF-treated cows but appear for the first time in control animals (Figure 5C, 6C, respectively). By 72 $\mathrm{h}$ postchallenge, control animals still showed increased surface expression of MPO (Figure 5D), but differences in monocyte MPO expression were no longer detectable (Figure 6D).
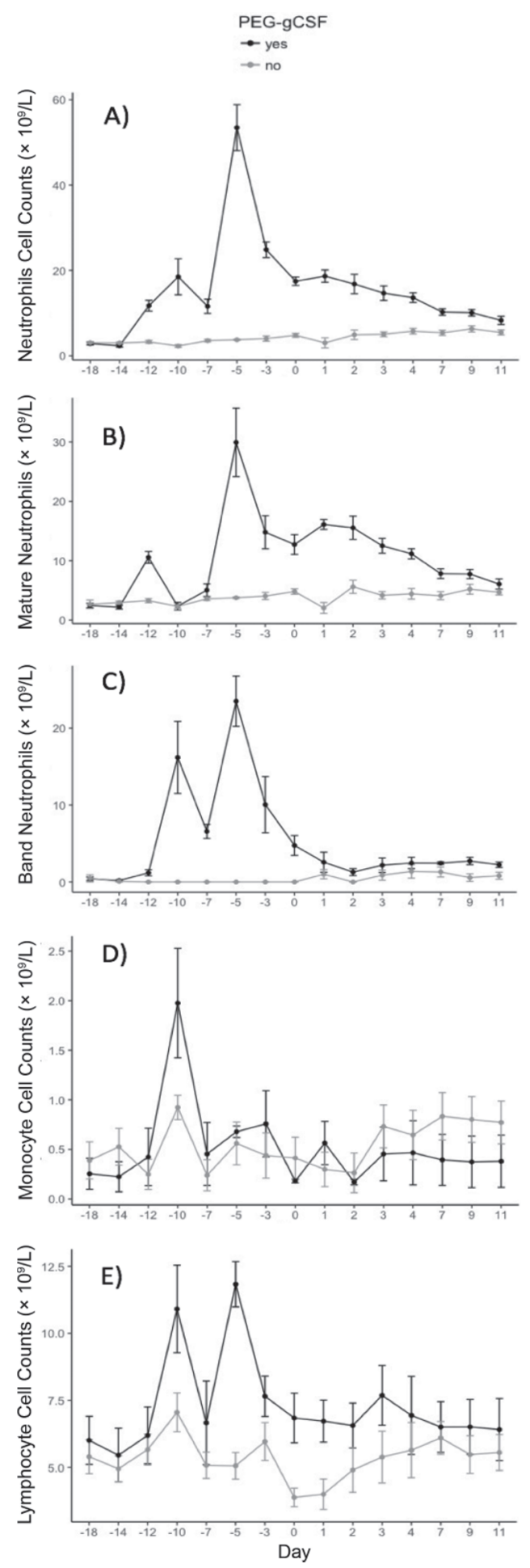

Figure 3. Effect of pegylated granulocyte colony-stimulating factor (PEG-gCSF) on immune cell profiles in whole blood. Cows were treated with PEG-gCSF after bleeds on $\mathrm{d}-14$ and again on $\mathrm{d}-7$ before challenge on d 0. Automated complete blood counts (CBC) produce (A) total neutrophil counts, which can be further analyzed by whole blood slide to determine proportion and counts of (B) mature neutrophils and $(\mathrm{C})$ band neutrophils. Automated CBC also revealed increasing circulating levels of monocytes (D), and lymphocytes (E). Error bars represent SE. 

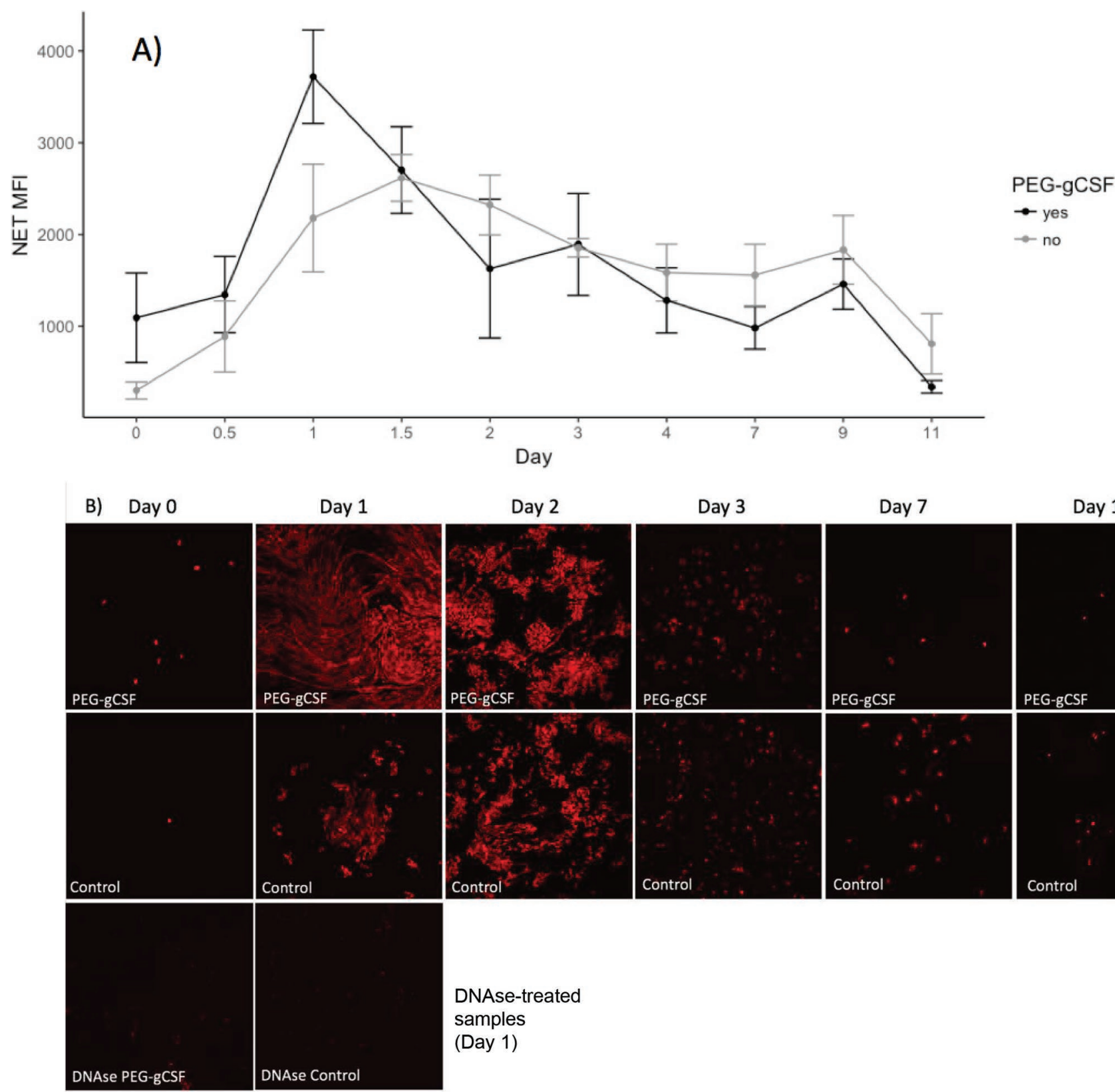

Figure 4. Functional assessment of neutrophils in milk examining neutrophil extracellular traps (NET). (A) mean fluorescent intensity (MFI) of Sytox-Orange (Invitrogen, Eugene, OR)-stained milk fat NET. (B) Confocal microscopy imaging of stained milk fat NET. Error bars represent SE. PEG-gCSF = pegylated granulocyte colony-stimulating factor.

\section{DISCUSSION}

The natural environment of the modern dairy cow and vast exposure to a diversity of pathogens makes the elimination of mastitis difficult. However, developing therapies, such as G-CSF treatment, provide a valuable tool in the management and prevention of bovine mastitis. We have shown that PEG-gCSF-treated Holsteins had reduced disease severity against an experimentally induced E. coli infection. This manuscript uniquely assesses mid-lactation subjects in contrast to the periparturient cow and establishes that PEG-gCSF-treated cows challenged with $E$. coli mastitis have reduced disease severity compared with nontreated controls. In addition to reduced bacterial counts and BSA levels, treated cows exhibited less reduced milk yield and less reduced feed intake during disease challenge. Although the majority of existing PEG-gCSF literature focuses on 

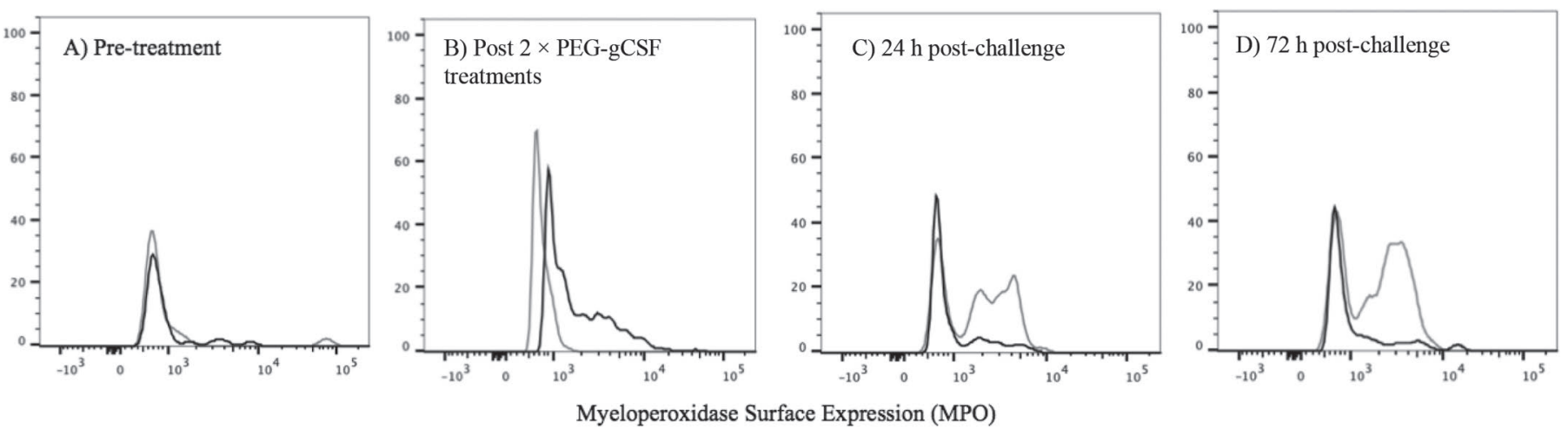

Figure 5. Myeloperoxidase (MPO) surface expression as an activation marker for blood neutrophils. Flow cytometry shows surface expression of MPO on neutrophils (A) before all pegylated granulocyte colony-stimulating factor (PEG-gCSF) treatments (d -14), (B) following both PEG-gCSF injections (d -5; peak activation), (C) $24 \mathrm{~h}$ postchallenge (d 1; peak infection), and (D) $72 \mathrm{~h}$ postchallenge (d 3 ; recovering from infection). Neutrophil cell populations from whole blood of representative PEG-gCSF (black) and control (gray) cows.

natural incidence of mastitis, our mid-lactation animals exhibit similar responses to what has been reported in earlier trials with regard to targeted neutrophil expansion.

Our data showed the second PEG-gCSF injection boosted neutrophil expansion as neutrophil numbers more than doubled in less time compared with the first initial injection (Figure 3A). Interestingly, hematology slide analysis shows that mature neutrophils respond initially to the first PEG-gCSF injection after $2 \mathrm{~d}$, whereas immature (band) cells have a delayed response peaking $4 \mathrm{~d}$ after the first treatment (Figure 3B, 3C). These observations may be illustrating a recruitment out of the bone marrow of granulocyte progenitor cells. The distinct behavior and relationships of immature and mature neutrophils with PEG-gCSF treatment in circulating blood may prove insightful to mechanisms of mastitis protection, cell maturation, and effector cell migration from the bone marrow to the site of inflam- mation. Uniquely, circulating monocytes only expand in cell number after the first PEG-gCSF injection (Figure 3D). It is possible that monocytes are involved in directing neutrophil behavior through various cell signals and suggests a potential role for monocyte activity in the initiation of a G-CSF response cascade. In addition, overall neutrophil numbers were higher in this study compared with those seen in previous studies. Our mid-lactation PEG-gCSF-treated animals averaged $53 \times 10^{9}$ neutrophils/L of blood compared with $18 \times 10^{9}$ neutrophils/L of blood at peak levels after 2 PEG-gCSF injections in previously published work (Canning et al., 2017; McDougall et al., 2017). This is likely due to the immune system differences between mid-lactation animals and periparturient immunesuppressed cows. Furthermore, the proportion of band neutrophils is much higher in our study than previously reported, with a greater number of band neutrophils compared with mature neutrophils after the first in-
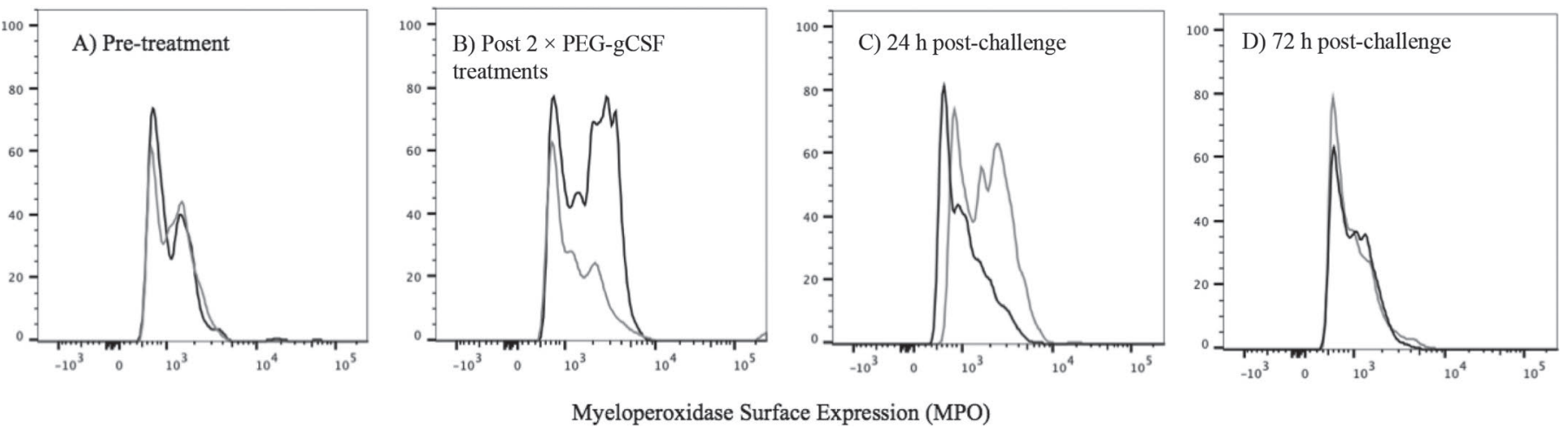

Myeloperoxidase Surface Expression (MPO)

Figure 6. Myeloperoxidase (MPO) surface expression as an activation marker for blood monocytes. Flow cytometry shows surface expression of MPO on monocytes (A) before all pegylated granulocyte colony-stimulating factor (PEG-gCSF) treatments (d -14), (B) following both PEG-gCSF injections (d -5; peak activation), (C) $24 \mathrm{~h}$ postchallenge (d 1; peak infection), and (D) $72 \mathrm{~h}$ postchallenge (d 3; recovering from infection). Monocyte cell populations from whole blood of representative PEG-gCSF (black) and control (gray) cows. 
jection and similar numbers after the second injection (Kimura et al., 2014). Our mid-lactation cows lack a target for neutrophil migration until we challenged them with mastitis and initiated an inflammation response in the mammary gland, resulting in higher neutrophil numbers and a different proportion of band and mature cells. By contrast, a periparturient cow may call activated neutrophils from the circulating blood stream to the uterus to compete with the increased presence of bacterial contaminants and to compensate for additional energy and immune requirements (Hammon et al., 2006; Sheldon et al., 2008). Neutrophil migration in response to microbial threat is well recognized, and impaired neutrophil function is correlated with metritis and additional uterine health disorders in the periparturient cow (Kimura et al., 2002; Hammon et al., 2006; Sheldon et al., 2008). A future study of interest might be to look at the effect of PEG-gCSF with experimental mastitis during the periparturient critical period.

The DNA, indicative of NET in milk fat in PEGgCSF-treated animals after infection, suggests that potential cell activation or migration signals were altered compared with negative controls. The increased presence of NET in the treated cow milk fat at $24 \mathrm{~h}$ postchallenge may be evidence of heighted systematic activation state of neutrophils (Figure 4). Alternatively, it is possible that the heightened activation of circulating neutrophils in the blood allows those cells to more rapidly respond to inflammation signals and appear more quickly in the milk and mammary gland in treated cows. The mechanism of differentiation and the relationship between different neutrophil subsets will continue to be investigated in the role of PEG-gCSF treatment. In addition to neutrophils, monocyte and lymphocyte populations also expand in response to the G-CSF cytokine treatment, which could contribute an important component of cytokine and cell signaling.

To assess cellular activation and the potential targeting of immune cells to the mammary gland, we used flow cytometric staining of surface molecules. In addition to its role in the production of antimicrobial reactive oxygen species, MPO has been identified as a ligand for E-selectin and cellular activation can cause its upregulated expression on the surface of monocytes and granulocytes in humans (Kindzelskii et al., 2006; Silvescu and Sackstein, 2014). We observed an increase in MPO surface expression after the first injection of PEG-gCSF (data not shown) with a peak MPO surface expression demonstrated after both PEG-gCSF injections in both neutrophils (Figure 5), and monocytes (Figure 6) in whole blood. Our hypothesis is that PEGgCSF treatment primes a population of neutrophils and monocytes to express surface MPO in the form of a cell adhesion molecule (E-selectin) ligand. Then, once the animals are challenged with an infectious agent, mammary epithelial cells would express E-selectin on their surfaces. This could allow the pre-primed neutrophils and monocytes faster entry into an infected gland, which could play a role in immune response and disease severity (Vandeputte-Van Messom et al., 1993). This hypothesis is supported by our observation of the MPO+ neutrophils and monocytes in blood in the PEG-gCSF animals before infection and the immediate loss of the MPO+ cells from circulation for treated cows upon challenge. In the normal untreated animals, MPO+ neutrophils and monocytes arise after infection perhaps to the natural generation of G-CSF, or general initiated inflammatory response to challenge. Also supporting the hypothesis that treated animals translocated immune cells from the circulation into the mammary gland is the elevated presence of NET in the milk fat of treated cows.

Despite the PEG-gCSF-induced dramatic increases in circulating neutrophils, one unanswered question is why we do not observe comparable increases in SCC in the mammary gland with disease challenge. From our analysis of mature versus immature neutrophils, we show that approximately half of our peak neutrophil numbers are immature band neutrophils likely being called recruited and differentiated from the bone marrow. One hypothesis that explains the lack of a massive increase in SCC in preinfected cows is that predominately mature neutrophils with upregulated surface adhesion molecules (e.g., MPO) efficiently migrate into the mammary gland (Van Merris et al., 2002). A possible explanation of why SCC are not different after infection could be that stimulated cells are so activated that when they enter the mammary gland, they quickly produce NET, in turn terminating the cell, and thus are uncountable in the SCC analysis. This was supported by the higher DNA concentrations and larger NET globules in PEG-gCSF cows immediately postchallenge (Figure 4).

With a commercially geared product intended for use across the dairy industry, we examined PEG-gCSF effects on SCC, milk yield, and feed intake under experimental infection conditions. Although raw SCC numbers did not identify significant differences between PEG-gCSF-treated and nontreated cows before or after infection (Figure 1A), it is likely this can be attributed to the large amount of variability and high standard errors from the SCC data. The log-transformed SCC data differed significantly with an increase from PEGgCSF-treated cows for the preinfection phase (Figure 1B). When we examine this observation, it is logical that a cytokine treatment targeting large circulating neutrophil expansion resulted in an increased SCC in the milk. However, the average raw SCC for the 
pretreatment phase were $\sim 86 \mathrm{~K} \mathrm{SCC}$ and $\sim 22 \mathrm{~K}$ SCC for the treated and untreated groups, respectively. Although the difference seen in the log-transformed data indicates an increase in SCC in the treated animals, the biological significance of a change of this magnitude is uncertain. It has been shown that there is more variation in repeatability of SCC data when counts are considered very low ( $<125 \mathrm{~K}$; Faust and Timms, 1995). Although it is unknown whether the increase of SCC or the increased number of "activated" circulating immune cells in PEG-gCSF-treated animals reduced the severity of the subsequent experimental infection, our hypothesis is that the priming of the immune system would be the major factor since the increase of SCC before infection was relatively small. Additional higher powered studies could be used to fully investigate the effect of PEG-gCSF on SCC numbers in healthy animals. It is also noteworthy that moderate increases in SCC in mid-lactation cows could differ from SCC responses in periparturient cows where immune and energy requirements are differently allocated.

Although all animals reduced milk yield as expected with disease challenge, the PEG-gCSF-treated animals maintained higher production volumes than control cows, suggesting reduced challenge stress and consistent with reduced disease severity (Figure 1C). Supportive of reduced stress on PEG-gCSF-treated animals were the similar results for feed intake (Figure 1D). This study focuses on animals under disease challenge, the serendipitous look at performance of cattle under the stress of silage feed change ( $d-2$ through $d 0)$ is also in agreement that PEG-gCSF treatment had no deleterious effects and may carry a slight advantage to treated cows under stress. Additional power and further experiments would be required to draw conclusions.

Collectively, PEG-gCSF-treated cows had reduced disease severity against an experimental mastitis challenge compared with nontreated controls. Treated cows had reduced bacterial counts and BSA levels, but also exhibit less reduced milk yield and less reduced feed intake under stress of challenge. Because the role of G-CSF seems to be to prime the immune system, this treatment is not implicated as a good candidate for use in an existing infection. The PEG-gCSF targeted a potent neutrophil expansion response both of mature and immature band neutrophils in circulation. Finally, PEG-gCSF treatment increase surface expression of a cell adhesion molecule (MPO) on neutrophils and monocytes, suggesting that these cells are primed for rapid egress from the circulation into a site of infection. These results continue to suggest a role for PEG-gCSF as an immune cell modulatory therapy for mastitis management and prevention.

\section{ACKNOWLEDGMENTS}

Special thanks go to Duane Zimmerman, Tera Nyholm, Adrienne Shircliff, Sam Humphrey, Randy Atchinson, and Denise Chapman (all at USDA, Ames, IA), Austin Putz (Iowa State University, Ames), and the USDA dairy care staff for their expert technical and animal handling expertise. Mention of trade names or commercial products in this article is solely for the purpose of providing specific information and does not imply recommendations or endorsement by the USDA. USDA is an equal opportunity provider and employer. The USDA was the sole funder for this research. The USDA determines the research priorities of all research that it funds. Publication of research is subject to review of USDA officials. Specific study design, data collection, data analysis, and preparation of the manuscript are under the control of the individual researchers.

\section{REFERENCES}

Brinkmann, V., U. Reichard, C. Goosmann, B. Fauler, Y. Uhlemann, D. S. Weiss, Y. Weinrauch, and A. Zychlinsky. 2004. Neutrophil extracellular traps kill bacteria. Science 303:1532-1535.

Burvenich, C., D. D. Bannerman, J. D. Lippolis, L. Peelman, B. J. Nonnecke, M. E. Kehrli Jr., and M. J. Paape. 2007. Cumulative physiological events influence the inflammatory response of the bovine udder to Escherichia coli infections during the transition period. J. Dairy Sci. 90(Suppl 1):E39-E54.

Cai, T. Q., P. G. Weston, L. A. Lund, B. Brodie, D. J. McKenna, and W. C. Wagner. 1994. Association between neutrophil functions and periparturient disorders in cows. Am. J. Vet. Res. 55:934-943.

Canning, P., R. Hassfurther, T. TerHune, K. Rogers, S. Abbott, and D. Kolb. 2017. Efficacy and clinical safety of pegbovigrastim for preventing naturally occurring clinical mastitis in periparturient primiparous and multiparous cows on US commercial dairies. J. Dairy Sci. 100:6504-6515.

Ceniti, C., D. Britti, A. M. L. Santoro, R. Musarella, L. Ciambrone, F. Casalinuovo, and N. Costanzo. 2017. Phenotypic antimicrobial resistance profile of isolates causing clinical mastitis in dairy animals. Ital. J. Food Saf 6:6612.

Cha, E., D. Bar, J. A. Hertl, L. W. Tauer, G. Bennett, R. N. Gonzalez, Y. H. Schukken, F. L. Welcome, and Y. T. Grohn. 2011. The cost and management of different types of clinical mastitis in dairy cows estimated by dynamic programming. J. Dairy Sci. 94:4476-4487.

Dorward, D. A., C. D. Lucas, A. L. Alessandri, J. A. Marwick, F. Rossi, I. Dransfield, C. Haslett, K. Dhaliwal, and A. G. Rossi. 2013. Technical advance: Autofluorescence-based sorting: Rapid and nonperturbing isolation of ultrapure neutrophils to determine cytokine production. J. Leukoc. Biol. 94:193-202.

Faust, M. A., and L. L. Timms. 1995. Estimates of variability for somatic cell count measurements in the Iowa dairy industry. J. Dairy Sci. 78:546-551.

Hammon, D. S., I. M. Evjen, T. R. Dhiman, J. P. Goff, and J. L. Walters. 2006. Neutrophil function and energy status in Holstein cows with uterine health disorders. Vet. Immunol. Immunopathol. 113:21-29.

Harp, J. A., M. E. Kehrli Jr., D. J. Hurley, R. A. Wilson, and T. C. Boone. 1991. Numbers and percent of $\mathrm{T}$ lymphocytes in bovine peripheral blood during the periparturient period. Vet. Immunol. Immunopathol. 28:29-35.

Heyneman, R., C. Burvenich, and R. Vercauteren. 1990. Interaction between the respiratory burst activity of neutrophil leukocytes and 
experimentally induced Escherichia coli mastitis in cows. J. Dairy Sci. 73:985-994.

Ismail, Z. B. 2017. Mastitis vaccines in dairy cows: Recent developments and recommendations of application. Vet. World 10:10571062 .

Kehrli, M. E., Jr., J. S. Cullor, and S. C. Nickerson. 1991a. Immunobiology of hematopoietic colony-stimulating factors: Potential application to disease prevention in the bovine. J. Dairy Sci. 74:4399-4412.

Kehrli, M. E., Jr., J. P. Goff, M. G. Stevens, and T. C. Boone. 1991b. Effects of granulocyte colony-stimulating factor administration to periparturient cows on neutrophils and bacterial shedding. J. Dairy Sci. 74:2448-2458.

Kimura, K., J. P. Goff, P. Canning, C. Wang, and J. A. Roth. 2014. Effect of recombinant bovine granulocyte colony-stimulating factor covalently bound to polyethylene glycol injection on neutrophil number and function in periparturient dairy cows. J. Dairy Sci. 97:4842-4851.

Kimura, K., J. P. Goff, M. E. Kehrli Jr., and T. A. Reinhardt. 2002. Decreased neutrophil function as a cause of retained placenta in dairy cattle. J. Dairy Sci. 85:544-550.

Kindzelskii, A. L., A. J. Clark, J. Espinoza, N. Maeda, Y. Aratani, R. Romero, and H. R. Petty. 2006. Myeloperoxidase accumulates at the neutrophil surface and enhances cell metabolism and oxidant release during pregnancy. Eur. J. Immunol. 36:1619-1628.

Lippolis, J. D., T. A. Reinhardt, J. P. Goff, and R. L. Horst. 2006. Neutrophil extracellular trap formation by bovine neutrophils is not inhibited by milk. Vet. Immunol. Immunopathol. 113:248-255.

Lippolis, J. D., T. A. Reinhardt, R. A. Sacco, B. J. Nonnecke, and C. D. Nelson. 2011. Treatment of an intramammary bacterial infection with 25-hydroxyvitamin D(3). PLoS One 6:e25479.

Mallard, B. A., J. C. Dekkers, M. J. Ireland, K. E. Leslie, S. Sharif, C. L. Vankampen, L. Wagter, and B. N. Wilkie. 1998. Alteration in immune responsiveness during the peripartum period and its ramification on dairy cow and calf health. J. Dairy Sci. 81:585-595.

McDougall, S., S. J. LeBlanc, and A. Heiser. 2017. Effect of prepartum energy balance on neutrophil function following pegbovigrastim treatment in periparturient cows. J. Dairy Sci. 100:7478-7492.

Paape, M. J., D. D. Bannerman, X. Zhao, and J. W. Lee. 2003. The bovine neutrophil: Structure and function in blood and milk. Vet. Res. 34:597-627.

Reinhardt, T. A., R. E. Sacco, B. J. Nonnecke, and J. D. Lippolis. 2013. Bovine milk proteome: Quantitative changes in normal milk exosomes, milk fat globule membranes and whey proteomes resulting from Staphylococcus aureus mastitis. J. Proteomics 82:141-154.
Revelo, X., A. L. Kenny, N. M. Barkley, and M. R. Waldron. 2011. Neutrophils harvested from the blood of dairy cows have impaired reactive oxygen species production and release of extracellular traps during the periparturient period. FASEB J. 25(1_supplement):38.38-38.38

Ruiz, R., L. O. Tedeschi, and A. Sepulveda. 2017. Investigation of the effect of pegbovigrastim on some periparturient immune disorders and performance in Mexican dairy herds. J. Dairy Sci. 100:33053317.

Ryman, V. E., G. M. Pighetti, J. D. Lippolis, J. C. Gandy, C. M. Applegate, and L. M. Sordillo. 2015. Quantification of bovine oxylipids during intramammary Streptococcus uberis infection. Prostaglandins Other Lipid Mediat. 121(Pt B):207-217.

Sheldon, I. M., E. J. Williams, A. N. Miller, D. M. Nash, and S. Herath. 2008. Uterine diseases in cattle after parturition. Vet. J. 176:115-121.

Sieff, C. A. 1987. Hematopoietic growth factors. J. Clin. Invest. 79:1549-1557.

Silvescu, C. I., and R. Sackstein. 2014. G-CSF induces membrane expression of a myeloperoxidase glycovariant that operates as an E-selectin ligand on human myeloid cells. Proc. Natl. Acad. Sci. USA 111:10696-10701.

Stabel, J. R., M. E. Kehrli Jr., J. R. Thurston, J. P. Goff, and T. C. Boone. 1991. Granulocyte colony-stimulating factor effects on lymphocytes and immunoglobulin concentrations in periparturient cows. J. Dairy Sci. 74:3755-3762.

USDA-APHIS. 2007. Highlights of Dairy2007 Part I: Reference of Dairy Health and Management in the United States. Pages 1-2.

Van Merris, V., E. Meyer, and C. Burvenich. 2002. Functional maturation during bovine granulopoiesis. J. Dairy Sci. 85:2859-2868.

Vandeputte-Van Messom, G., C. Burvenich, E. Roets, A. M. MassartLeen, R. Heyneman, W. D. Kremer, and A. Brand. 1993. Classification of newly calved cows into moderate and severe responders to experimentally induced Escherichia coli mastitis. J. Dairy Res. 60:19-29

Xu, S., M. Hoglund, L. Hakansson, and P. Venge. 2000. Granulocyte colony-stimulating factor (G-CSF) induces the production of cytokines in vivo. Br. J. Haematol. 108:848-853.

Yeiser, E. E., K. E. Leslie, M. L. McGilliard, and C. S. Petersson-Wolfe. 2012. The effects of experimentally induced Escherichia coli mastitis and flunixin meglumine administration on activity measures, feed intake, and milk parameters. J. Dairy Sci. 95:4939-4949. 